\title{
Effects of melatonin on colonic anastomosis healing following chemotherapy in rats
}

\author{
Cebrail $\underline{A k y u z}^{1}$, MD, Necdet Fatih $\underline{\text { Yasar }}^{2}, \mathrm{MD}$, Orhan $\underline{\text { Uzun }}{ }^{2}, \mathrm{MD}$, KIvanc Derya Peker ${ }^{2}$, MD, Oguzhan Sunamak ${ }^{1}$, MD,
} Mustafa Duman' ${ }^{2}$, MD, Ahmet Ozer Sehirli ${ }^{3}, \mathrm{PhD}, \operatorname{Sinan} \underline{\mathrm{Yol}}^{4}, \mathrm{MD}$

INTRODUCTION This study aimed to investigate the effects of melatonin on the healing of colon anastomosis following chemotherapy.

METHODS 32 rats were randomised into four groups: (a) control group, which underwent sigmoid colon transection and primary anastomosis; (b) melatonin group, which received melatonin daily following anastomosis; (c) 5-fluorouracil (5-FU) group, which received 5-FU for five days prior to anastomosis; and (d) 5-FU+melatonin group, which received 5-FU for five days prior to anastomosis and melatonin daily following anastomosis. The rats were sacrificed on Postoperative Day 7 and anastomotic bursting pressures were measured. The anastomotic segment was extracted for hydroxyproline, luminol and lucigenin measurement and histopathological examination. Blood samples were obtained from the vena cava for measurement of tumour necrosis factor-alpha (TNF- $\alpha$ ) and interleukin-1 beta (IL-1 $\beta$ ) plasma levels.

RESULTS Compared to the 5-FU group, bursting pressures of anastomosis and hydroxyproline levels were significantly higher, while luminol and lucigenin levels were significantly lower, in the control and 5-FU+melatonin groups. In addition, TNF- $\alpha$ and IL- $1 \beta$ plasma levels were significantly lower in the control and 5-FU+melatonin groups than in the 5-FU group. Histopathological examination showed a significant decrease in inflammation and necrosis formation in the melatonin group when compared to the control group. The positive effect of melatonin was also seen in the rats that received 5-FU. CONCLUSION Our study results showed that the adverse effects of chemotherapy on the mechanical, biochemical and histopathological parameters of anastomosis healing were attenuated through melatonin treatment.

Keywords: 5-fluorouracil, colonic anastomosis, healing, melatonin, oxidative stress

\section{INTRODUCTION}

Anastomotic leakage is an important cause of morbidity and mortality secondary to colorectal surgery. The risk of colorectal anastomotic dehiscence is greater when compared to other parts of the gastrointestinal tract because of the higher bacterial content and scant blood supply of the colon, especially in chemotherapy patients. ${ }^{(1-3)}$ Colorectal anastomotic dehiscence is correlated with prolonged length of hospital stay and increased treatment cost. ${ }^{(4-6)}$ The rate of anastomotic leakage after colorectal surgery is $2 \%-37 \%$ in the English-language literature. ${ }^{(7-9)}$

Anastomotic complications are related to technical, local and systemic factors, which can affect the healing period. Absence of tension on the suture line, perfusion of intestinal edges, ability to ensure homeostasis and absence of distal luminal obstruction are important for safe anastomosis. ${ }^{(10-12)}$ Risk factors for anastomotic dehiscence include malnutrition, anaemia, local infections, obesity, female gender, intraoperative hypotension, emergent surgery and disrupted blood supply, as well as preoperative neoadjuvant therapies. ${ }^{(10,11,13-19)}$

Melatonin has been reported to have favourable effects on wound healing. ${ }^{(20)}$ However, it is unclear whether melatonin can alleviate the potential side effects of preoperative chemotherapy on wound healing. The aim of the present study was to investigate the effects of melatonin on the mechanical, biochemical and histopathological parameters of anastomosis healing after preoperative chemotherapy.

\section{METHODS}

This study was approved by the Local Ethical Committee of Animal Experiments of Marmara University (protocol no. 73.2012.mar). In this study, 32 adult male Wistar rats weighing 200-250 g were used. All animals were kept in a temperature-controlled room $\left(22^{\circ} \mathrm{C} \pm 2{ }^{\circ} \mathrm{C}\right)$ with a 12 -hour light-dark cycle and had free access to water and standard laboratory chow.

5-fluorouracil (5-FU) was administered at a dose of $20 \mathrm{mg} / \mathrm{kg}$ daily for five days before surgery, while melatonin was administered subcutaneously at a dose of $0.4 \mathrm{mg} / \mathrm{kg}$ daily following surgery. Rats were randomised into four groups: (a) control group, with colonic anastomosis without administration of 5-FU or melatonin; (b) melatonin group, with colonic anastomosis and melatonin; (c) 5-FU group, with colonic anastomosis and 5-FU; and (d) 5-FU+melatonin group, with colonic anastomosis and administration of both 5-FU and melatonin.

All rats were fasted for 12 hours prior to the surgical procedure. Anaesthesia was induced by administration of $50 \mathrm{mg} / \mathrm{kg}$ ketamine (Ketalarß; Pfizer, Ortakoy, Istanbul, Turkey) and $10 \mathrm{mg} / \mathrm{kg}$ xylazine hydrochloride (Rompun®; Bayer, Umraniye, Istanbul, Turkey). Abdominal hair was shaved, skin was sterilised with

\footnotetext{
${ }^{1}$ Department of Surgery, Haydarpasa Numune Training and Research Hospital, ${ }^{2}$ Department of Surgery, Koşuyolu High Specialized Training and Research Hospital, ${ }^{3}$ Department of Pharmacology, Faculty of Dentistry, Near East University, ${ }^{4}$ Department of General and Gastroenterological Surgery, Faculty of Health Sciences, Istanbul Medeniyet University, İstanbul, Turkey

Correspondence: Dr Cebrail Akyuz, Instructor, Department of Surgery, Haydarpasa Numune Training And Research Hospital, Tıbbiye Cad. No: 23, 34668, Usküdar/Istanbul, Turkey.drcakyuz@hotmail.com
} 
$10 \%$ povidone iodine, and laparotomy via a midline incision was performed under general anaesthesia. The colon was transected without disrupting perfusion of the mesocolon. Afterwards, anastomosis was performed using interrupted $6 / 0$ polypropylene (Prolene®; Ethicon, Johnson \& Johnson Medical NV, Belgium) sutures in a single-layer, end-to-end manner. All anastomoses were performed by a single surgeon and eight sutures were used for each one. Fascia and skin were closed using continuous 3/0 polypropylene (Prolene ${ }^{\circledR}$; Ethicon). Standard laboratory chow and water was given 12 hours after the procedure. Rats were sacrificed on Postoperative Day 7 and the peritoneal cavity was exposed with a midline incision.

The anastomotic colonic parts were resected en bloc with $2 \mathrm{~cm}$ of bowel on each side. To measure bursting pressure, the segments were infused ( $6 \mathrm{~mL} / \mathrm{min}$ ) with saline containing methylene blue via infusion pump, and the outcome measure was expressed in $\mathrm{mmHg}$. After measuring bursting pressure, a $5 \mathrm{~mm}$-wide ring of tissue, including the anastomosis, was removed. Half of this removed tissue was wrapped in aluminium foil and preserved at $-20^{\circ} \mathrm{C}$ for later measurements of hydroxyproline, luminol and lucigenin levels, while the other half was fixed in $10 \%$ formaldehyde for histopathological evaluation. The amount of hydroxyproline was determined following the methods used by Jamall et al. ${ }^{(21)}$

To assess the contribution of reactive oxygen species in 5-FU-induced colonic damage, luminol and lucigenin chemiluminescence was measured as indicators of radical formation. Measurements were performed at room temperature using a portable tube luminometer (Junior LB 9509 luminometer; EG\&G Berthold, Bad Wildbad, Germany). Specimens were put into vials containing phosphate-buffered saline (PBS)/HEPES (4-[2-hydroxyethyl]-1-piperazineethanesulfonic acid) buffer (0.5 M PBS containing 20 mM HEPES, pH 7.2). Reactive oxygen species were quantified after the addition of enhancers, lucigenin or luminol, to a final concentration of $0.2 \mathrm{mM}$. Luminol detects a group of reactive oxygen species (i.e. hydroxyl $[\bullet \mathrm{OH}]$, hydrogen peroxide $\left[\mathrm{H}_{2} \mathrm{O}_{2}\right]$ and hypochlorous acid $[\mathrm{HOCl}]$ radicals), while lucigenin is selective for the superoxide radical $\left(\bullet \mathrm{O}_{2}{ }^{-}\right) \cdot{ }^{(22,23)}$ Counts were obtained at one-minute intervals, and the results were given as area under the curve for a counting period of five minutes. Counts were corrected for wet tissue weights and expressed as relative light units (RLU/mg tissue). ${ }^{(24)}$

Plasma levels of tumour necrosis factor-alpha (TNF- $\alpha$ ) and interleukin-1 beta (IL-1 $\beta$ ) were quantified according to the manufacturer's instructions and guidelines using enzyme-linked immunosorbent assay kits specific to the previously mentioned rat cytokines (BioSource International, Nivelles, Belgium). The total antioxidant capacity in plasma was measured by using the colourimetric test system (ImAnOx, catalogue no. KC5200, Immundiagnostik AG, Bensheim, Hessen, Germany), according to the instructions provided by the manufacturer. These particular assay kits were selected because of their high degree of sensitivity, specificity, inter- and intra-assay precision, and the small amount of plasma sample required to conduct the assay.

For light microscopic analysis, samples from the colon were fixed in $10 \%$ buffered formalin for 48 hours, dehydrated in ascending alcohol series and embedded in paraffin wax. Sections with a thickness of approximately $5 \mathrm{~mm}$ were stained with haematoxylin and eosin for general morphology. For scanning electron microscopic investigation, the samples were fixed in $4 \%$ phosphate-buffered glutaraldehyde $(0.13 \mathrm{M}, \mathrm{pH} 7.4)$ for four hours and post-fixed with $1 \%$ osmium tetroxide for one hour, dehydrated in graded alcohol series, put into amyl acetate, dried with liquid carbon dioxide under pressure with a critical point dryer (Bio-Rad E 3000; Bio-Rad Laboratories, Clinical Diagnostics Distributor, Şahinbey, Gaziantep, Turkey) and covered with gold particles (Bio-Rad SC502; Bio-Rad Laboratories, Clinical Diagnostics Distributor, Şahinbey, Gaziantep, Turkey). Sections were observed under a photomicroscope (Olympus $\mathrm{BH} 2$; Olympus Optical Co Ltd, Tokyo, Japan) or a scanning electron microscope (SEM; Jeol Ltd 7200 JSM, Tokyo, Japan) by an experienced histologist who was unaware of the experimental groups.

Histopathological and biochemical observations were made by a histopathologist and biochemist who were blinded to the groups. Statistical analyses were performed using PASW Statistics for Windows version 18.0 (SPSS Inc, Chicago, IL, USA). The results were analysed using Kruskal-Wallis one-way analysis of variance with Tukey test. A p-value $<0.05$ was considered to be statistically significant.

\section{RESULTS}

During the trial, the site of bursting was outside the anastomosis line in four rats: one in the control group, one in the melatonin group and two in the 5-FU group. These rats were excluded from the study. In the following days, new trials were held in the same conditions and rat numbers of all groups were raised to eight.

All parameters in the control and melatonin groups were similar except for hydroxyproline levels, which were higher in the melatonin group than in the control group $(p<0.01$; Table I). Bursting pressure in the 5 -FU group was significantly lower when compared to the control $(p<0.01)$ and $5-F U+$ melatonin $(p<0.05)$ groups. Hydroxyproline levels in the 5 -FU group were significantly lower than in the control and 5 -FU+melatonin groups $(\mathrm{p}<0.05$ for both). Luminol levels in the 5-FU group were significantly higher than in the control and 5 -FU+melatonin groups $(p<0.001$ for both). Lucigenin levels in the 5-FU group were significantly higher than in the control $(p<0.001)$ and $5-\mathrm{FU}+$ melatonin $(p<0.01)$ groups. Serum TNF- $\alpha$ levels in the 5 -FU group were significantly higher than in the control and 5-FU+melatonin groups $(\mathrm{p}<0.05$ for both). Likewise, serum IL-1 $\beta$ levels in the 5 -FU group were significantly higher than in the control and $5-\mathrm{FU}+$ melatonin groups ( $p<0.05$ for both).

On histopathological assessment, the inflammation that was seen along the incision line in the control group (Fig. 1) was observed to be significantly decreased in the melatonin group (Fig. 2). In the 5-FU group, the incision line was not distinct, and severe inflammation and necrosis was observed (Fig. 3). Administration of melatonin resulted in morphological distinction of the incision line and regression of inflammation (Fig. 4). 
Table I. Mechanical and biochemical parameters of anastomosis healing.

\begin{tabular}{|lllll|}
\hline Variable & \multicolumn{3}{c|}{ Group (mean \pm standard error) } \\
\cline { 2 - 5 } & Control & Melatonin & 5-FU & 5-FU+melatonin \\
\hline Anastomotic bursting pressure $(\mathbf{m m H g})$ & $135.40 \pm 8.70$ & $165.00 \pm 2.90$ & $104.40 \pm 4.40^{*}$ & $126.40 \pm 4.30^{\S}$ \\
\hline Tissue level & & & & \\
\hline Hydroxyproline $(\mathrm{mg} / \mathrm{g}$ protein) & $0.55 \pm 0.06$ & $0.98 \pm 0.13^{*}$ & $0.23 \pm 0.03^{+}$ & $0.49 \pm 0.06^{\S}$ \\
\hline Luminol chemiluminescence (RLU/mg) & $124.40 \pm 11.90$ & $104.40 \pm 15.10$ & $244.70 \pm 27.50^{\ddagger}$ & $120.00 \pm 13.90^{* * *}$ \\
\hline Lucigenin chemiluminescence (RLU/mg) & $135.50 \pm 6.30$ & $109.20 \pm 5.50$ & $308.00 \pm 33.60^{\ddagger}$ & $186.50 \pm 15.20^{* *}$ \\
\hline Plasma level & & & & $67.20 \pm 4.30^{+}$ \\
\hline TNF- $\alpha$ assay $(\mathrm{pg} / \mathrm{mL})$ & $52.90 \pm 2.90$ & $44.10 \pm 2.70$ & $54.10 \pm 2.40^{\S}$ \\
\hline IL-1 $\beta$ assay $(\mathrm{pg} / \mathrm{mL})$ & $361 \pm 22$ & $323 \pm 16$ & $460 \pm 30^{+}$ & $365 \pm 11^{\S}$ \\
\hline
\end{tabular}

Compared to control group, ${ }^{*} p<0.01,+p<0.05, \neq p<0.001$. Compared to 5-FU group, $\$ p<0.05, p<0.001,{ }^{* *} p<0.01 .5$-FU: 5-fluorouracil; IL-1 $\beta$ : interleukin-1 beta; RLU relative light unit; TNF- $\alpha$ : tumour necrosis factor-alpha

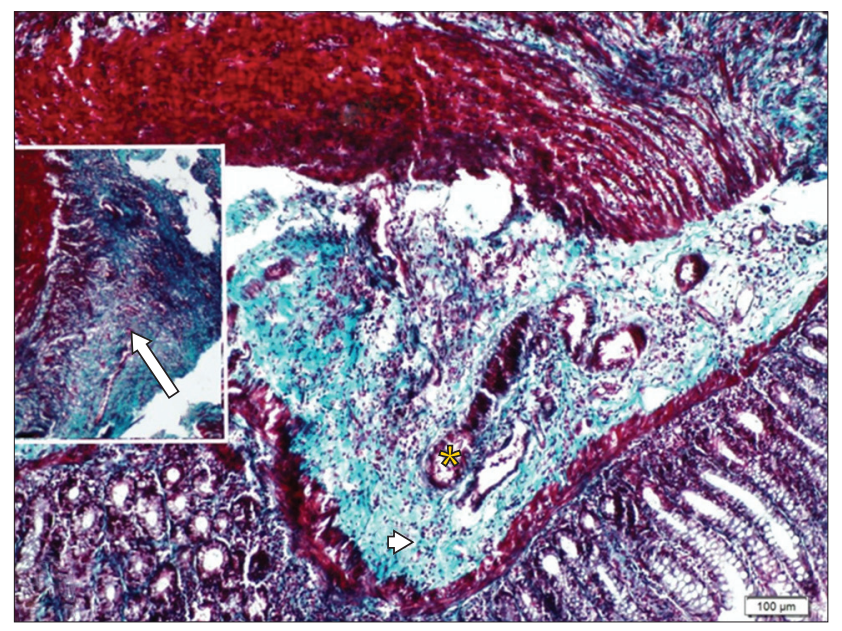

Fig. 1 Photomicrograph of control group rat specimen shows severe inflammation in the lamina propria (arrow), polymorphonuclear leucocytes (arrowhead) and capillary vessels $\left({ }^{*}\right)$ (Haematoxylin \& eosin, $\times 10$ ).

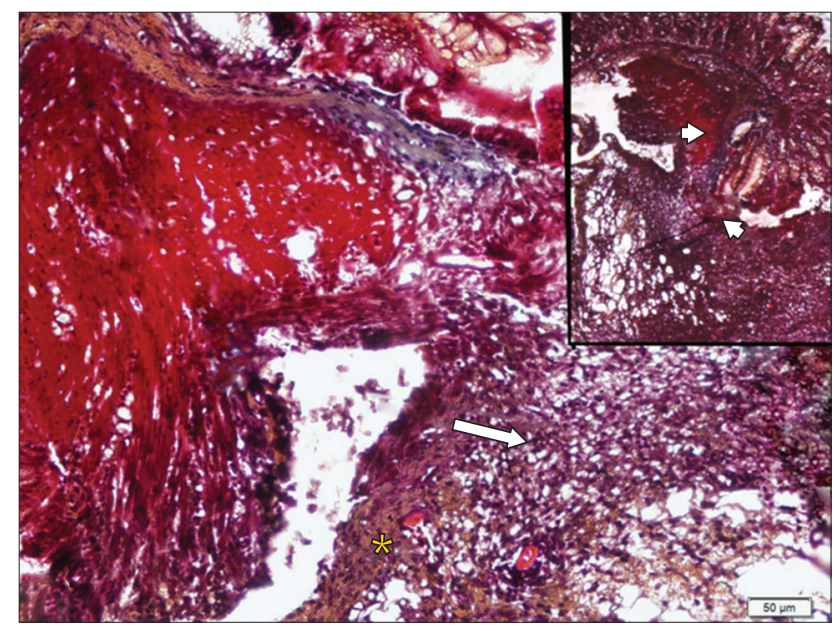

Fig. 3 Photomicrograph of 5 -fluorouracil group rat specimen shows anastomosis line (arrowheads) alongside the lamina propria, with inflammation (arrow) and necrosis $\left(^{*}\right)$ (Haematoxylin \& eosin, $\times 20$ ).

\section{DISCUSSION}

Our main finding was that the adverse effects of preoperative 5-FU administration on colonic anastomosis can be attenuated by postoperative melatonin treatment. Anastomosis leakage in colorectal surgery is an important cause of morbidity and mortality. ${ }^{(25,26)}$ There have been many clinical and experimental

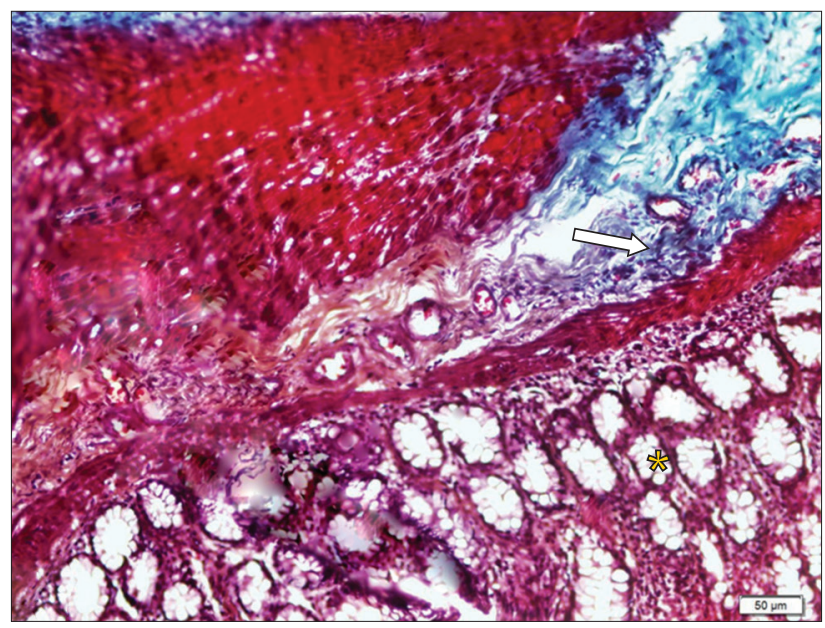

Fig. 2 Photomicrograph of melatonin group rat specimen shows regressed inflammation in the lamina propria (arrow) and gland structure ${ }^{*}$ ) (Haematoxylin \& eosin, $\times 20$ ).

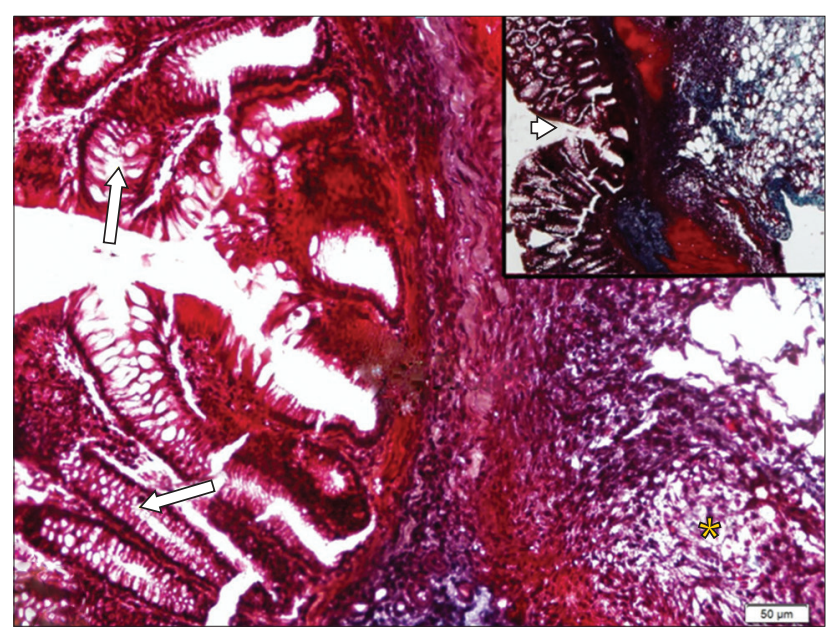

Fig. 4 Photomicrograph of 5-fluorouracil and melatonin group rat specimen shows apparent anastomosis line (arrowhead), apparent epithelial structure (arrows) and regressed inflammation $\left({ }^{*}\right)$ (Haematoxylin \& eosin, $\times 20$ ).

trials investigating the effects of surgical procedures or adjuvant therapy modalities on wound healing and anastomosis leakage. The adverse effects of preoperative neoadjuvant therapies on anastomosis are controversial. While many authors have suggested that preoperative neoadjuvant therapies do not affect the integrity of anastomosis, others proposed that colonic healing 
may be impaired when preoperative therapies are applied, as we have shown in our study. ${ }^{(27-33)}$

The findings of previous studies about the effect of melatonin on wound healing are also controversial. Drobnik and Dabrowski reported that pinealectomy induced elevation of collagen content in wounds, while dose-dependent melatonin suppressed this effect. $^{(34)}$ In another study about the effects of pinealectomy on wound healing, Bulbuller et al found that melatonin administration delayed wound healing and decreased collagen synthesis. ${ }^{(35)}$ In contrast to these findings, other studies have suggested that melatonin administration increased collagen synthesis and had a positive effect on wound healing. Pugazhenthi et al showed that melatonin decreased the amount of inducible nitric oxide synthase enzyme, increased the synthesis of angiogenesis and collagen, and thus accelerated wound healing in a rat model. ${ }^{(20)}$ Therefore, in the present study, we aimed to investigate the effects of melatonin on colonic anastomosis in rats that were preoperatively treated with $5-\mathrm{FU}$.

Bursting pressure indicates growing anastomotic strength, as long as disruption occurs within the anastomotic area in the first week following surgery for the bursting pressure. ${ }^{(36-38)}$ Biert et al identified that the bursting site was mostly outside the suture line in their control group, while treatment with postoperative 5-FU led to weakening of the anastomosis, as indicated by a shift in the bursting site to the anastomotic area and reduced mean values. ${ }^{(31)}$ Bozdag et al showed that preoperative treatment with intraperitoneal 5-FU can impair colonic healing in the anastomosis line, as indicated by reduced mean values of anastomotic bursting pressure. ${ }^{(33)}$ In our study, bursting pressure was measured on Postoperative Day 7 and bursting outside the anastomosis line was observed in four rats - one in the control group, one in the melatonin group and two in the 5-FU group. Bursting pressure in the 5-FU group was significantly lower when compared to the control group and this decrease was mitigated by melatonin treatment.

One of the methods for evaluation of anastomotic healing is analysis of hydroxyproline levels, which is an important indicator of collagen synthesis. ${ }^{(36,39)}$ Kuzu et al reported that anastomotic hydroxyproline content was significantly decreased after 5-FU therapy. ${ }^{(30)}$ However, these authors did not observe any effect on the clinical outcome of colonic anastomosis. On the other hand, Biert et al found that anastomotic hydroxyproline levels were higher in rats that received preoperative irradiation and postoperative 5-FU. ${ }^{(31)}$ In our study, hydroxyproline levels were significantly decreased in the 5-FU group, and this effect of 5-FU was prevented by the administration of melatonin.

Reactive oxygen species radicals arise from inflammation and immune-mediated injury. ${ }^{(40,41)}$ Luminol detects the $\mathrm{H}_{2} \mathrm{O}_{2}, \bullet \mathrm{OH}$, hypochlorite $\left(\mathrm{OCl}^{-}\right)$, peroxynitrite $\left(\mathrm{OONO}^{-}\right)$and lipid peroxyl radicals, while lucigenin is selective for $\bullet \mathrm{O}_{2}{ }^{-}$radicals. ${ }^{(24)}$ To the best of our knowledge, no study in the literature has investigated luminol and lucigenin levels at the anastomosis line. In our study, free oxygen radical levels were measured in the colon anastomosis tissue using the chemiluminescence method. We found that luminol and lucigenin levels were significantly higher in the 5-FU group than in the control group. However, melatonin treatment prevented this oxidative stress caused by preoperative 5-FU therapy.

TNF- $\alpha$ and IL- $1 \beta$ have an important role in the regulation of inflammation and their cytotoxic effects. ${ }^{(42)}$ Neutrophils secrete free oxygen radicals in inflammation and trauma and a set of lysosomal enzymes as well as cytokines, such as TNF- $\alpha$ and IL- $1 \beta$, when they are stimulated. ${ }^{(43)}$ In our study, serum TNF- $\alpha$ and IL-1 $\beta$ levels were increased in the 5-FU group when compared to the control group, and were significantly decreased after melatonin treatment. This showed that the activation and infiltration of neutrophils, which play a precipitating role in tissue damage, had been inhibited by melatonin and consequently resulted in a decreased inflammatory response.

Kuzu et al, who investigated the effects of chemotherapy and radiotherapy on colonic anastomotic healing by evaluating histopathological parameters, determined that inflammatory exudates and necrosis persist while granulation tissue formation is delayed after such protocols. ${ }^{(44)}$ In our study, the incision line was histopathologically distinct and necrosis was not detected in the melatonin group. Inflammatory cell infiltration in the anastomosis line and decrease in neutrophils were identified following melatonin treatment. This histopathological finding showed that melatonin has a positive effect on colonic anastomotic healing. In the 5-FU group, the incision line was not distinct, and a severe increase in inflammation and necrotic areas was identified. It was observed that melatonin therapy following administration of 5-FU resulted in a distinct incision line, and regression of inflammation and necrosis.

In conclusion, our study suggests that preoperative chemotherapy has negative effects on colonic anastomotic healing. These detrimental effects of chemotherapy on the mechanical, biochemical and histopathological parameters of anastomosis healing may be attenuated by postoperative melatonin therapy.

\section{REFERENCES}

1. Rolandelli RH, Koruda MJ, Settle RG, Rombeau JL. Effects of intraluminal infusion of short-chain fatty acids on the healing of colonic anastomosis in the rat. Surgery 1986; 100:198-204.

2. Hesp WL, Lubbers EJ, de Boer HH, Hendriks T. Anastomotic insufficiency in small bowel surgery--incidence and treatment. Langenbecks Arch Chir 1986; 368:105-11.

3. Schrock TR, Deveney CW, Dunphy JE. Factor contributing to leakage of colonic anastomoses. Ann Surg 1973; 177:513-8.

4. Mäkelä JT, Kiviniemi H, Laitinen S. Risk factors for anastomotic leakage after left-sided colorectal resection with rectal anastomosis. Dis Colon Rectum 2003; 46:653-60.

5. Akyol AM, McGregor JR, Galloway DJ, Murray GD, George WD. Anastomotic leaks in colorectal cancer surgery: a risk factor for recurrence? Int J Colorectal Dis 1991; 6:179-83.

6. Petersen S, Freitag M, Hellmich G, Ludwig K. Anastomotic leakage: impact on local recurrence and survival in surgery of colorectal cancer. Int J Colorectal Dis 1998; 13:160-3.

7. Braskén P. Healing of experimental colon anastomosis. Eur J Surg Suppl 1991; (566): $1-51$.

8. Konishi T, Watanabe T, Kishimoto J, Nagawa H. Risk factors for anastomotic leakage after surgery for colorectal cancer: results of prospective surveillance. J Am Coll Surg 2006; 202:439-44.

9. Hyman NH, Osler T, Cataldo P, Burns EH, Shackford SR. Anastomotic leaks after bowel resection: what does peer review teach us about the relationship to postoperative mortality? J Am Coll Surg 2009; 208:48-52.

10. Curley SA, Allison DC, Smith DE, Doberneck RC. Analysis of techniques and results in 347 consecutive colon anastomoses. Am J Surg 1988; 155:597-601. 
11. Mantovani M, Leonardi LS, Gomes de Alcântara F, et al. [Comparative study of different types of sutures in single or double layers in the small intestine. Experimental study in dogs]. AMB Rev Assoc Med Bras 1976; 22:79-86. Portuguese.

12. Jex RK, van Heerden JA, Wolff BG, Ready RL, Ilstrup DM. Gastrointestinal anastomoses. Factors affecting early complications. Ann Surg 1987; 206:138-41.

13. Franca A, Ramalho FS, Ramalho LN, da Rocha JJ, Féres O. Effects of preoperative pelvic irradiation on colonic anastomosis healing. An experimental study in rats. Acta Cir Bras 2008; 23 Suppl 1:24-30.

14. Alves A, Panis $Y$, Trancart D, et al. Factors associated with clinically significan anastomotic leakage after large bowel resection: multivariate analysis of 707 patients. World J Surg 2002; 26:499-502.

15. Pucciarelli S, Toppan P, Friso ML, et al. Preoperative combined radiotherapy and chemotherapy for rectal cancer does not affect early postoperative morbidity and mortality in low anterior resection. Dis Colon Rectum 1999; 42:1276-84.

16. Golub R, Golub RW, Cantu R Jr, Stein HD. A multivariate analysis of factors contributing to leakage of intestinal anastomoses. J Am Coll Surg 1997; 184:364-72.

17. Rullier E, Laurent $\mathrm{C}$, Garrelon JL, et al. Risk factors for anastomotic leakage after resection of rectal cancer. Br J Surg 1998; 85:355-8.

18. Feres O, Monteiro dos Santos Jr JC, Andrade JI. The role of mechanical bowel preparation for colonic resection and anastomosis: an experimental study. Int J Colorectal Dis 2001; 16:353-6.

19. Alberts JC, Parvaiz A, Moran BJ. Predicting risk and diminishing the consequences of anastomotic dehiscence following rectal resection. Colorecta Dis 2003; 5:478-82.

20. Pugazhenthi K, Kapoor M, Clarkson AN, Hall I, Appleton I. Melatonin accelerates the process of wound repair in full-thickness incisional wounds. J Pineal Res 2008; 44:387-96.

21. Jamall IS, Finelli VN, Que Hee SS. A simple method to determine nanogram levels of 4-hydroxyproline in biological tissues. Anal Biochem 1981; 112:70-5.

22. Davies GR, Simmonds NJ, Stevens TR, et al. Mucosal reactive oxygen metabolite production in duodenal ulcer disease. Gut 1992; 33:1467-72.

23. Ohara Y, Peterson TE, Harrison DG. Hypercholesterolemia increases endothelial superoxide anion production. J Clin Invest 1993; 91:2546-51.

24. Haklar G, Yüksel M, Yalçin AS. Chemiluminescence in the measurement of free radicals: theory and application on a tissue injury model. Marmara Med J 1998; 11:56-60.

25. Ptok H, Marusch F, Meyer F, et al. Impact of anastomotic leakage on oncological outcome after rectal cancer resection. Br J Surg 2007; 94:1548-54.

26. Khan AA, Wheeler JM, Cunningham $C$, et al. The management and outcome of anastomotic leaks in colorectal surgery. Colorectal Dis 2008; 10:587-92.

27. El-Malt M, Ceelen W, van den Broecke C, et al. Healing of experimental colonic anastomoses: effects of combined preoperative high-dose radiotherapy and intraperitoneal 5-fluorouracil. Int J Cancer 2001; 96:297-304.
28. Ersoy E, Akbulut H, Moray G. Effects of oxaliplatin and 5-fluorouracil on the healing of colon anastomoses. Surg Today 2009; 39:38-43.

29. Ozel L, Ozel MS, Toros AB, et al. Effect of early preoperative 5-fluorouracil on the integrity of colonic anastomoses in rats. World J Gastroenterol 2009; 15:4156-62.

30. Kuzu MA, Köksoy C, Kale T, Demirpençe E, Renda N. Experimental study of the effect of preoperative 5-fluorouracil on the integrity of colonic anastomoses. Br J Surg 1998; 85:236-9.

31. Biert J, Wobbes T, Hoogenhout J, de Man B, Hendriks T. Combined preoperative irradiation and direct postoperative 5-fluorouracil without negative effects on early anastomotic healing in the rat colon. Radiother Oncol 1996; 41:257-62.

32. Blouhos K, Pramateftakis MG, Tsachalis $\mathrm{T}$, et al. The integrity of colonic anastomoses following the intraperitoneal administration of oxaliplatin. Int J Colorectal Dis 2010; 25:835-41.

33. Bozdag AD, Peker $Y$, Derici H, Gürkök $C$, Ozgönül $M$. The effect of preoperative 5-fluorouracil on colonic healing: an experimental study. Hepatogastroenterology 2001; 48:1631-4.

34. Drobnik J, Dabrowski R. Melatonin suppresses the pinealectomy-induced elevation of collagen content in a wound. Cytobios 1996; 85:51-8.

35. Bulbuller N, Dogru O, Yekeler H, et al. Effect of melatonin on wound healing in normal and pinealectomized rats. J Surg Res 2005; 123:3-7.

36. Hendriks T, Mastboom WJ. Healing of experimental intestinal anastomoses. Parameters for repair. Dis Colon Rectum 1990; 33:891-901.

37. Kiyama T, Onda M, Tokunaga A, Efron DT, Barbul A. Effect of matrix metalloproteinase inhibition on colonic anastomotic healing in rats. J Gastrointest Surg 2001; 5:303-11.

38. Månsson $\mathrm{P}$, Zhang XW, Jeppsson B, Thorlacius H. Anastomotic healing in the rat colon: comparison between a radiological method, breaking strength and bursting pressure. Int J Colorectal Dis 2002; 17:420-5.

39. Koruda MJ, Rolandelli RH. Experimental studies on the healing of colonic anastomoses. J Surg Res 1990; 48:504-15.

40. Grisham MB, Volkmer C, Tso P, Yamada T. Metabolism of trinitrobenzene sulfonic acid by the rat colon produces reactive oxygen species. Gastroenterology $1991 ; 101: 540-7$

41. Siems WG, Grune T, Werner A, et al. Protective influence of oxypurinol on the trinitrobenzene sulfonic acid (TNB) model of inflammatory bowel disease in rats. Cell Mol Biol 1992; 38:189-99.

42. Semenzato G. Tumour necrosis factor: a cytokine with multiple biological activities. Br J Cancer 1990; 61:354-61.

43. Hillegass LM, Griswold DE, Brickson B, Albrightson-Winslow C. Assessment of myeloperoxidase activity in whole rat kidney. J Pharmacol Methods 1990; 24:285-95

44. Kuzu MA, Kuzu I, Köksoy C, et al. Histological evaluation of colonic anastomotic healing in the rat following preoperative 5-fluorouracil, fractionated irradiation, and combined treatment. Int J Colorectal Dis 1998; 13:235-40. 